\title{
ABSORÇÃO E UTILIZAÇÃO DO FÓSFORO PELAS CULTURAS DA SOJA E DO FEIJÃO E POR PLANTAS DANINHAS ${ }^{(1)}$
}

\author{
Sergio de Oliveira Procópio ${ }^{(2)}$, José Barbosa dos Santos ${ }^{(3)}$, \\ Fábio Ribeiro Pires ${ }^{(2)}$, Antonio Alberto da \\ Silva $^{(4)} \&$ Eduardo de Sá Mendonça ${ }^{(5)}$
}

\begin{abstract}
RESUMO
A aplicação de fertilizantes pode, em determinadas situações, beneficiar mais as plantas daninhas do que as próprias culturas. $O$ manejo de fertilizantes em sistemas agrícolas pode ser um importante componente em programas de manejo integrado das culturas. $O$ objetivo deste trabalho foi avaliar a eficiência na absorção e utilização do $P$ pelas culturas da soja e do feijão e por espécies de plantas daninhas. $O$ experimento foi realizado em casa de vegetação, no período de agosto a novembro de 2002. O delineamento experimental utilizado foi o de blocos casualizados com três repetições; os tratamentos originaram-se de esquema fatorial $6 \times 4$, sendo seis espécies vegetais: soja (Glycine $\max$ ), feijão (Phaseolus vulgaris), dois biótipos de Euphorbia heterophylla (suscetível e resistente aos herbicidas inibidores de ALS), Bidens pilosa e Desmodium tortuosum; e quatro doses de $\mathrm{P}\left(0,0012,00 ; 24,00\right.$ e $\left.48,00 \mathrm{mg} \mathrm{dm}^{-3}\right)$, aplicadas na semeadura sob a forma de superfosfato simples. A soja foi a espécie que revelou maior aumento na sua massa seca de raízes com o incremento do fornecimento de $\mathrm{P}$. D. tortuosum, soja e B. pilosa apresentaram maior resposta à adição de doses crescentes de $\mathbf{P}$ em relação ao acúmulo de massa seca. $O$ maior teor de $\mathbf{P}$ foi constatado para o feijão, independente da dose de P aplicada, contudo $D$. tortuosum foi a única espécie a ter seu teor de $\mathrm{P}$ aumentado em quase três vezes, quando cultivado com o dobro da dose aplicada $\left(48,00 \mathrm{mg} \mathrm{dm}^{-3}\right)$, com base nas necessidades da cultura do feijão. Até a avaliação realizada no início do florescimento, as plantas de soja e $D$. tortuosum foram as espécies que
\end{abstract}

\footnotetext{
(1) Recebido para publicação em abril de 2004 e aprovado em março de 2005.

(2) Professor do Departamento de Agronomia, Universidade de Rio Verde - FESURV. Faz. Fontes do Saber, Depto. de Agronomia. Caixa Postal 104, CEP 75901-970 Rio Verde (GO). E-mail: soprocopio@yahoo.com.br; frpires@yahoo.com.br

${ }^{(3)}$ Doutorando do Departamento de Fitotecnia, Universidade Federal de Viçosa - UFV. Av. PH Rolfs s/n; CEP 36571-000 Viçosa (MG). E-mail: jbarbosasantos@yahoo.com.br

(4) Professor do Departamento de Fitotecnia, UFV. E-mail: aasilva@ufv.br

(5) Professor do Departamento de Solos, UFV. E-mail:esm@ufv.br
} 
acumularam a maior quantidade de $P$ em seus tecidos, porém, durante a fase reprodutiva foi $D$. tortuosum a qual, juntamente com feijão, apresentaram variações quanto à eficiência de suas raízes em absorver $\mathrm{P}$, de acordo com $\mathbf{a}$ dose desse nutriente, enquanto as demais espécies mantiveram a mesma eficiência radicular, independente do teor de $\mathbf{P}$ do solo. A eficiência na utilização do $\mathbf{P}$ absorvido, observado para $D$. tortuosum, soja e feijão, diminuiu com o aumento da dose. Nos biótipos de E. heterophylla e no feijão, foi verificado baixo desempenho quanto ao uso eficiente do $\mathrm{P}$ disponível no solo. A maior porcentagem de recuperação do $\mathrm{P}$ aplicado até à fase de formação de propágulos foi constatada nas plantas de $D$. tortuosum.

Termos de indexação: Glycine max, Phaseolus vulgaris, Bidens pilosa, Euphorbia heterophylla, Desmodium tortuosum.

\title{
SUMMARY: ABSORPTIONAND USE OF PHOSPHORUS BY SOYBEANAND COMMON BEAN CROPS AND BY WEEDS
}

\begin{abstract}
This work aimed to evaluate the absorption and use of phosphorus $(P)$ by soybean and common bean crops and by weeds. The experiment was carried out in a greenhouse from August to November 2002. I was adopted a randomized block design with three replications, in a $6 \times 4$ factorial scheme. Six vegetal species (soybean (Glycine max), common bean (Phaseolus vulgaris), two Euphorbia heterophylla biotypes (susceptible and resistant to $A L S$ inhibitor herbicides), Bidens pilosa and Desmodium tortuosum) and four doses of $P$ (0.00 12.00; 24.00 and $\left.48.00 \mathrm{mg} \mathrm{dm}^{-3}\right)$, applied at sowing in the form of single super phosphate, were evaluated. The soybean root biomass was increased by the higher $\mathrm{P}$ supply and soybean, D. tortuosum, and $\mathrm{B}$. pilosa accumulated more biomass in response to the increasing $P$ doses. The highest $P$ concentration was observed in the bean plants, independent of the applied P level, while D. tortuosum was the only specie in which the P content nearly tripled when cultivated with a $P$ dose $\left(48.00 \mathrm{mg} \mathrm{dm}^{-3}\right)$ that was twice as high as that recommended for common bean. The highest $P$ content in the plant tissues was accumulated by soybean and $\mathrm{D}$. tortuosum until the beginning of flowering, and by $\mathrm{D}$. tortuosum during the reproductive phase. In relation to the $P$ uptake efficiency of roots, D. tortuosum and common bean presented variations according to the applied $P$ level. The other species maintained the same root efficiency, independent of the Pavailability in the soil. The efficiency in the use of absorbed P by D. tortuosum, soybean and common bean decreased with the increase of the $P$ dose. The $\mathrm{E}$. heterophylla biotypes and common bean performed worst in relation to the efficient use of available $P$ in the soil. Until the reproductive phase, D. Tortuosum presented the highest percentage of recovery of applied $P$.
\end{abstract}

Index terms: Glycine max, Phaseolus vulgaris, Bidens pilosa, Euphorbia heterophylla, Desmodium tortuosum.

\section{INTRODUÇÃO}

A aplicação de fertilizantes, especialmente os macronutrientes como o $\mathrm{P}$, com vistas em aumentar a produção das culturas agrícolas é completamente aceita e comprovada (Radosevich et al., 1997). Contudo, Tomaso (1995) e Patterson (1995) reportam que a aplicação de fertilizantes, freqüentemente, beneficia mais as plantas daninhas do que as próprias culturas. Um mecanismo descrito por Patterson (1995) evidencia que muitas plantas daninhas em áreas com boa disponibilidade de nutrientes acumulam-nos em concentrações acima da necessária para o seu desenvolvimento. Isso indica que não é correto corrigir deficiências nutricionais das culturas, provocadas pela competição com plantas daninhas, simplesmente pelo incremento nas aplicações de fertilizantes (Radosevich et al., 1997).

O manejo de fertilizantes em sistemas agrícolas pode constituir importante componente em programas de manejo integrado de plantas daninhas (Blackshaw et al., 2003). Os fertilizantes podem ser usados para alterar as relações de competitividade, de modo a favorecer as espécies cultivadas, desde que as espécies competidoras apresentem respostas diferenciadas à aplicação de nutrientes (Armstrong et al., 1993). A aplicação de fertilizantes fosfatados 
em áreas agrícolas pode influenciar a mudança da comunidade e a densidade populacional de plantas daninhas infestantes (Gilbert \& Pember, 1975; Hoveland et al., 1976). Armstrong et al. (1993) constataram que a adição de $\mathrm{P}$ não só ocasionou incremento no crescimento e na absorção de nutrientes de plantas de Cenchrus ciliaris (espécie desejável), mas também aumentou a habilidade competitiva dessa espécie contra a invasora Aristida armata. Por outro lado, Vengris et al. (1955) verificaram maior redução na produção de milho associado a plantas daninhas, em vasos que receberam fertilizante fosfatado, quando comparados aos que não a receberam. Também Cralle et al. (2003) observaram que o crescimento de plantas de trigo foi menos inibido em solo com deficiência de $\mathrm{P}$ do que o crescimento da planta daninha Lolium multiflorum, cultivada neste mesmo solo. Shafiq et al. (1994) constataram que a aplicação de $\mathrm{P}$ não diminuiu o grau de competição entre plantas daninhas e a espécie cultivada Vigna radiata.

Vários estudos relatam os efeitos negativos das plantas daninhas quanto à competição por $\mathrm{P}$ com as plantas cultivadas. Ponce et al. (1996) observaram que a absorção de $\mathrm{P}$ por plantas de tomate apresentou queda de 1,37 para $0,90 \mathrm{~g}_{\text {planta }}{ }^{-1} \mathrm{de} \mathrm{P}$, quando estas foram submetidas à competição com Solanum nigrum. Santos et al. (1998) descreveram que a presença de Amaranthus hybridus em competição com alface ocasionou aumento do conteúdo total de $\mathrm{P}$ nessa planta daninha e diminuição nas plantas de alface. Do mesmo modo, Santos et al. (1998) relataram que plantas de Portulaca oleracea sob competição com plantas de alface apresentaram aumento do teor de $\mathrm{P}$, em relação ao monocultivo dessa planta daninha.

Tradicionalmente, a eficiência de utilização de um nutriente tem sido definida como a razão entre a matéria seca total produzida e a quantidade total do nutriente na matéria seca (Pereira, 1992). Segundo Siddiqi \& Glass (1981), essa eficiência de utilização do nutriente é tão importante quanto a eficiência de absorção. A eficiência de absorção pode ser definida como o P total na planta / P aplicado (Youngquist et al., 1992), enquanto a eficiência de utilização de $\mathrm{P}$, considerando as raízes, foi descrita por Jones et al. (1992) como P absorvido/matéria seca das raízes. Armstrong et al. (1993) concluíram que Cenchrus ciliaris apresentou maior eficiência no uso do P do que Aristida armata, Digitaria ammophila e Thyridolepis mitchelliana.

O objetivo deste trabalho foi avaliar a eficiência na absorção e utilização do $\mathrm{P}$ pelas culturas da soja e do feijão e por plantas daninhas normalmente encontradas nas áreas agrícolas do País, visando adquirir subsídios para o estabelecimento de estratégias quanto ao fornecimento de nutrientes que favoreçam as culturas em detrimento das plantas daninhas.

\section{MATERIAL E MÉTODOS}

O experimento foi realizado em casa de vegetação entre novembro de 2001 e março de 2002. O delineamento experimental utilizado foi o de blocos casualizados com três repetições. Os tratamentos originaram-se de esquema fatorial $6 \times 4$, sendo seis espécies vegetais [soja (Glycine max cv. Capinópolis), feijão (Phaseolus vulgaris cv. Pérola), Euphorbia heterophylla susceptível aos herbicidas inibidores da enzima acetolactato sintase (ALS), Euphorbia heterophylla resistente aos herbicidas inibidores de ALS, Bidens pilosa e Desmodium tortuosum] e quatro doses de $\mathrm{P}_{2} \mathrm{O}_{5}$ : sem adição $\left(0,0 \mathrm{~kg} \mathrm{ha}^{-1}\right)$, metade da dose recomendada $\left(55,0 \mathrm{~kg} \mathrm{ha}^{-1}\right)$, dose recomendada $\left(110,0 \mathrm{~kg} \mathrm{ha}^{-1}\right)$ e duas vezes a dose recomendada $\left(220,0 \mathrm{~kg} \mathrm{ha}^{-1}\right)$ equivalentes a 0,00 ; 12,$00 ; 24,00$ e $48,00 \mathrm{mg} \mathrm{dm}^{-3}$ de P, respectivamente, misturado ao solo antes da semeadura, sob a forma de superfosfato simples $\left(20 \%\right.$ de $\left.\mathrm{P}_{2} \mathrm{O}_{5}\right)$. Foram utilizados dois vasos por bloco para cada tratamento, sendo um avaliado no início do florescimento e o outro no estádio final da formação de propágulos.

Os nutrientes $\mathrm{K}$ e $\mathrm{N}$ foram fornecidos para todos os tratamentos em mesma quantidade, sendo o $\mathrm{K}$ aplicado misturado ao solo, antes da semeadura, e o $\mathrm{N}$ em cobertura 20 e 40 dias após a semeadura (DAS). A quantidade dos nutrientes fornecidos (NPK) foi determinada com base na cultura do feijão para o nível tecnológico 4 (Chagas et al., 1999) e na análise do solo (Quadro 1).

Foram utilizados vasos plásticos que continham $3 \mathrm{dm}^{3}$ de solo. As amostras de solo foram destorroadas, homogeneizadas, secas ao ar e passadas em peneira de malha de $2 \mathrm{~mm}$. Em cada vaso foram semeadas cinco sementes, para as culturas, e 20 sementes, para as plantas daninhas. Após o crescimento das plantas, foi realizado o desbaste, deixando-se apenas uma planta por vaso. Para a manutenção da umidade do solo, foram feitas três irrigações diárias.

Nas épocas de avaliação, as plantas foram coletadas, tendo sido a massa seca dividida em parte aérea e raízes. Após esta etapa, a matéria vegetal fresca de raízes e parte aérea foi colocada em estufa com circulação forçada de ar a $75^{\circ} \mathrm{C}$, para secagem até peso constante. Posteriormente, as amostras do tecido vegetal foram moídas em moinho tipo Wiley, de aço inoxidável, passadas em peneira de 20 mesh $(0,85 \mathrm{~mm})$, acondicionadas em sacos de papel, para serem analisadas quimicamente.

Para determinar os teores de $\mathrm{P}$, as amostras do material vegetal foram submetidas à digestão nítrico-perclórica (proporção 4:1), sendo os teores de $\mathrm{P}$ determinados por colorimetria pelo método azul de molibdênio, tendo ácido ascórbico como agente redutor (Braga \& Defelipo, 1974). 
Quadro 1. Composição físico-química do Argissolo Vermelho-Amarelo utilizado no experimento

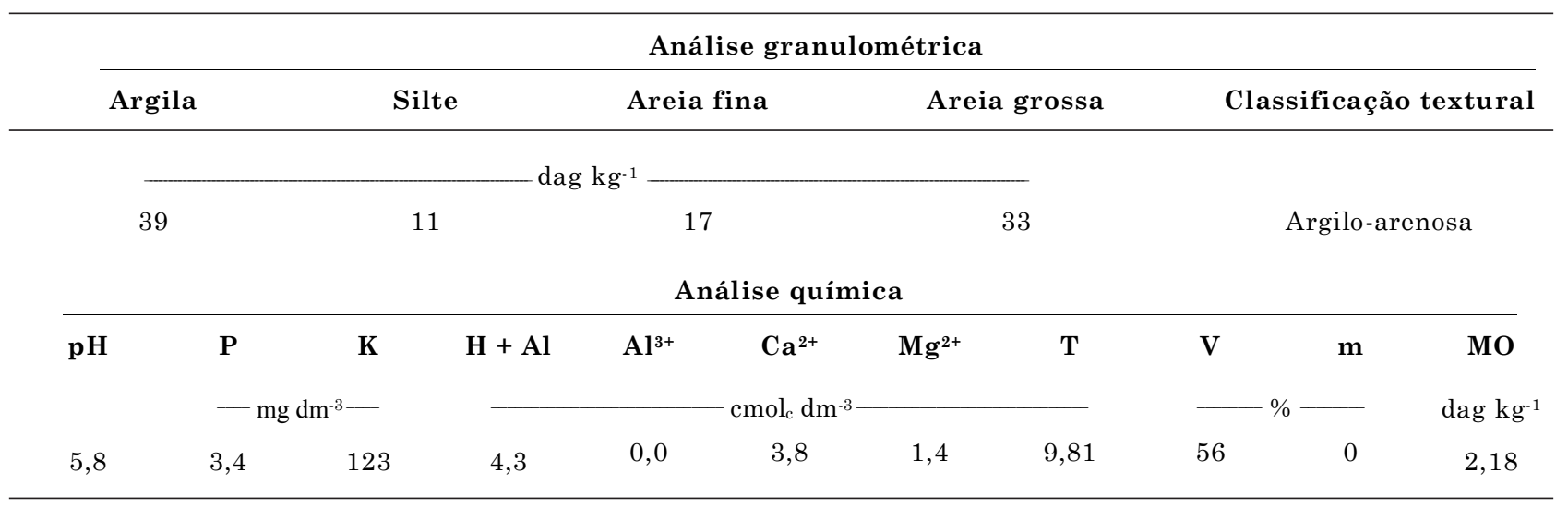

Após coleta e tabulação dos dados, foram calculados os seguintes índices de eficiência de absorção e utilização do P:

a) Eficiência das raízes na absorção de P (ERAP): conteúdo total de P na planta (mg) / massa seca de raízes (g planta ${ }^{-1}$ ).

b) Eficiência da utilização do $\mathrm{P}$ absorvido (EUPa): massa seca total (g planta $\left.{ }^{-1}\right) /$ conteúdo total de P na planta (mg).

c) Uso eficiente do $\mathrm{P}$ disponível, $\left(\mathrm{UEP}_{\mathrm{d}}\right)$ : massa seca total (g planta $\left.{ }^{-1}\right) / \mathrm{P}$ disponível no solo $\left(\mathrm{mg}\right.$ vaso $\left.^{-1}\right)$. Para determinar o $\mathrm{P}$ disponível no solo, efetuouse análise do solo, nas duas épocas de avaliação, em vasos que continham as mesmas adições para cada teor de P; no entanto, sem cultivo durante todo o período.

d) Percentagem de recuperação do $\mathrm{P}$ aplicado (PRPA): [conteúdo de P na planta fertilizada (mg) - conteúdo P na testemunha (mg)] x 100 / quantidade de $\mathrm{P}$ aplicado $\left(\mathrm{mg} \mathrm{vaso}^{-1}\right)$, via fertilizante.

Os dados obtidos foram submetidos à análise de variância. Os efeitos entre as espécies vegetais, dentro de cada dose do nutriente, foram avaliados pelo teste de Tukey a $5 \%$. Os efeitos entre doses do nutriente dentro de cada planta foram analisados por meio de análise de regressão, sendo os modelos de regressão escolhidos com base na significância dos coeficientes da equação pelo teste $t$, no coeficiente de determinação $\left(\mathrm{R}^{2}\right)$ e na adequação biológica.

\section{RESULTADOS E DISCUSSÃO}

De acordo com a análise da produção de massa seca de raízes (MSR), apresentada pelas espécies vegetais coletadas no início do florescimento e no final da formação de propágulos, a soja apresentou maior MSR em comparação ao feijão em todos os níveis aplicados de P (Quadro 2). Quando não se aplicou $\mathrm{P}$ ao solo, o maior valor de MSR no início do florescimento foi verificado para as plantas de soja, apenas não diferindo da espécie daninha $D$. tortuosum, a qual, nesse período de avaliação, só apresentou MSR menor, em relação à soja, na dose de 12,00 $\mathrm{mg} \mathrm{dm}^{-3}$ (Quadro 2).

Dentre as plantas daninhas, os biótipos de $E$. heterophylla foram as espécies que produziram a menor MSR, em ambos os períodos avaliados, não se observando diferenças quanto à produção de MSR entre os biótipos (Quadro 2). Observou-se que, na avaliação realizada no final da formação de propágulos, o feijão apresentou menor MSR do que as plantas daninhas $B$. pilosa e $D$. tortuosum para todos os níveis de $\mathrm{P}$, o que pode caracterizar desvantagem da cultura em competir por água e nutrientes. Pelo fato de o $\mathrm{P}$ ser considerado nutriente de baixa mobilidade no solo, plantas de maior sistema radicular apresentam vantagem na captura desse elemento (Santos et al., 1998). Todas as espécies responderam à adição de $\mathrm{P}$ quanto à produção de MSR (Quadro 3); todavia, as maiores respostas foram observadas nas espécies soja, $D$. tortuosum e B. pilosa.

Na produção de massa seca total (MST), assim como na avaliação do acúmulo de MSR, a soja foi superior ao feijão em todas as doses de $\mathrm{P}$ e nas duas épocas de avaliação (Quadro 4). Martins \& Pitelli (2000) observaram aumento de 208,5 \% na massa seca de plantas de soja, com a aplicação de $200 \mathrm{mg} \mathrm{dm}^{-3}$ de P. No início do florescimento, $D$. tortuosum apresentou a maior MST entre as plantas daninhas, com exceção da dose de $\mathrm{P}$ de 24,00 mg dm ${ }^{-3}$, e não diferiu da MST acumulada por plantas de $B$. pilosa. Contudo, na avaliação realizada no final da 
Quadro 2. Massa seca de raízes (MSR) das culturas do feijão e da soja e de espécies de plantas daninhas, submetidas a quatro níveis de fósforo na semeadura

\begin{tabular}{|c|c|c|c|c|c|c|c|c|}
\hline \multirow{4}{*}{ Espécie } & \multicolumn{8}{|c|}{ MSR } \\
\hline & \multicolumn{4}{|c|}{ Início do florescimento } & \multicolumn{4}{|c|}{ Final da formação de propágulos } \\
\hline & \multicolumn{8}{|c|}{ Dose de P $\left(\mathrm{mg} \mathrm{dm}^{-3}\right)$} \\
\hline & $\mathbf{0 , 0 0}$ & 12,00 & 24,00 & 48,00 & $\mathbf{0 , 0 0}$ & 12,00 & 24,00 & 48,00 \\
\hline & \multicolumn{8}{|r|}{-} \\
\hline P. vulgaris & $0,39 \mathrm{bc}$ & $0,73 \mathrm{bc}$ & $1,08 \mathrm{bc}$ & $1,86 \mathrm{c}$ & $0,65 \mathrm{c}$ & $1,32 \mathrm{c}$ & $1,73 \mathrm{c}$ & $2,15 \mathrm{~d}$ \\
\hline G. $\max$ & $1,49 \mathrm{a}$ & $2,32 \mathrm{a}$ & $2,77 \mathrm{a}$ & $4,60 \mathrm{a}$ & $2,60 \mathrm{a}$ & $5,24 \mathrm{a}$ & $7,19 \mathrm{a}$ & $10,15 \mathrm{a}$ \\
\hline E. heterophylla $(\mathrm{r})^{(1)}$ & $0,14 \mathrm{bc}$ & $0,30 \mathrm{c}$ & $0,22 \mathrm{c}$ & $1,17 \mathrm{c}$ & $1,22 \mathrm{bc}$ & $1,47 \mathrm{c}$ & $2,19 \mathrm{c}$ & $3,70 \mathrm{c}$ \\
\hline E. heterophylla $(\mathrm{s})^{(1)}$ & $0,06 \mathrm{c}$ & $0,43 \mathrm{bc}$ & $0,28 \mathrm{c}$ & $1,18 \mathrm{c}$ & $0,94 \mathrm{c}$ & $1,21 \mathrm{c}$ & $2,17 \mathrm{c}$ & $2,65 \mathrm{~cd}$ \\
\hline B. pilosa & $0,43 \mathrm{bc}$ & $0,61 \mathrm{bc}$ & $1,82 a b$ & $3,06 \mathrm{~b}$ & $3,25 \mathrm{a}$ & $4,11 \mathrm{ab}$ & $6,54 \mathrm{a}$ & $7,75 \mathrm{~b}$ \\
\hline D. tortuosum & $1,16 \mathrm{ab}$ & $1,32 \mathrm{~b}$ & $1,99 a b$ & $4,23 \mathrm{a}$ & $3,63 \mathrm{a}$ & $3,68 \mathrm{~b}$ & $5,29 \mathrm{~b}$ & $8,65 \mathrm{~b}$ \\
\hline
\end{tabular}

(1) Biótipo resistente e sensível aos herbicidas inibidores da enzima ALS. Médias seguidas de letras iguais, na coluna, não diferem entre si pelo teste de Tukey a $5 \%$.

Quadro 3. Equações de regressão da massa seca de raízes (MSR), massa seca total (MST) e da quantidade acumulada de fósforo nas plantas (PPLANTA) das culturas do feijão e da soja e de espécies de plantas daninhas, em resposta a doses (D) de $P$

\begin{tabular}{|c|c|c|c|c|c|}
\hline Espécie & MSR (Início do florescimento) & $\mathbf{R}^{2}$ & \multicolumn{2}{|c|}{ MSR (Final da formação de propágulos) } & $\mathbf{R}^{2}$ \\
\hline P. vulgaris & $\hat{\mathrm{Y}}=0,37+0,0102 \mathrm{X}$ & 0,99 & $\hat{\mathrm{Y}}$ & $=0,8326+0,0100 \mathrm{X}$ & 0,92 \\
\hline G. $\operatorname{Max}$ & $\hat{\mathrm{Y}}=1,45+0,0213 \mathrm{X}$ & 0,95 & $\hat{\mathrm{Y}}$ & $=3,0674+0,0513 \mathrm{X}$ & 0,98 \\
\hline E. heterophylla $(\mathrm{r})^{(1)}$ & $\hat{\mathrm{Y}}=0,14+0,007 \mathrm{X}$ & 0,82 & $\hat{\mathrm{Y}}$ & $=1,0204+0,0179 \mathrm{X}$ & 0,97 \\
\hline E. heterophylla $(\mathrm{s})^{(1)}$ & $\hat{Y}=0,028+0,0073 X$ & 0,85 & $\hat{\mathrm{Y}}$ & $=0,9526+0,0125 \mathrm{X}$ & 0,92 \\
\hline B. pilosa & $\hat{\mathrm{Y}}=0,254+0,0195 \mathrm{X}$ & 0,96 & $\hat{\mathrm{Y}}$ & $=3,349+0,0327 \mathrm{X}$ & 0,93 \\
\hline \multirow[t]{2}{*}{ D. tortuosum } & $\hat{Y}=0,782+0,0222 X$ & 0,93 & $\hat{\mathrm{Y}}$ & $=2,077+0,0455 \mathrm{X}$ & 0,99 \\
\hline & MST (Início do florescimento) & & \multicolumn{2}{|c|}{ MST (Final da formação de propágulos) } & \\
\hline P. vulgaris & $\hat{\mathrm{Y}}=1,116+0,0225 \mathrm{X}$ & 0,99 & $\hat{\mathrm{Y}}$ & $=3,446+0,0529 \mathrm{X}$ & 0,91 \\
\hline G. $\max$ & $\hat{\mathrm{Y}}=4,706+0,0609 \mathrm{X}$ & 0,99 & $\hat{\mathrm{Y}}$ & $=9,5628+0,1646 \mathrm{X}$ & 0,98 \\
\hline E. heterophylla $(\mathrm{r})^{(1)}$ & $\hat{\mathrm{Y}}=0,11+0,0145 \mathrm{X}$ & 0,82 & $\hat{\mathrm{Y}}$ & $=3,4044+0,0927 \mathrm{X}$ & 0,96 \\
\hline E. heterophylla $(\mathrm{s})^{(1)}$ & $\hat{\mathrm{Y}}=0,11+0,0173 \mathrm{X}$ & 0,89 & $\hat{\mathrm{Y}}$ & $=3,4202+0,0855 \mathrm{X}$ & 0,97 \\
\hline B. pilosa & $\hat{\mathrm{Y}}=0,496+0,0465 \mathrm{X}$ & 0,97 & $\hat{\mathrm{Y}}$ & $=10,9958+0,1648 \mathrm{X}$ & 0,98 \\
\hline \multirow[t]{2}{*}{ D. tortuosum } & $\hat{\mathrm{Y}}=4,938+0,0807 \mathrm{X}$ & 0,99 & $\hat{\mathrm{Y}}$ & $=13,8756+0,1520 \mathrm{X}$ & 0,91 \\
\hline & $\begin{array}{l}\text { P planta (Início do } \\
\text { florescimento) }\end{array}$ & & \multicolumn{2}{|c|}{$\begin{array}{c}\text { P planta (Final da formação de } \\
\text { propágulos) }\end{array}$} & \\
\hline P. vulgaris & $\hat{\mathrm{Y}}=1,344+0,0615 \mathrm{X}$ & 0,95 & $\hat{\mathrm{Y}}$ & $=8,1096+0,1636 \mathrm{X}$ & 0,99 \\
\hline G. $\max$ & $\hat{Y}=8,16+0,1628 \mathrm{X}$ & 0,98 & $\hat{\mathrm{Y}}$ & $=7,045+0,2270 \mathrm{X}$ & 0,97 \\
\hline E. heterophylla $(\mathrm{r})^{(1)}$ & $\begin{array}{l}\hat{Y} \\
\hat{Y}\end{array}=0,268+0,0286 \mathrm{X}$ & 0,84 & $\begin{array}{l}\hat{Y} \\
\hat{\mathbf{Y}}\end{array}$ & $=5,5424+0,1424 \mathrm{X}$ & 0,98 \\
\hline E. heterophylla $(\mathrm{s})^{(1)}$ & $\hat{\mathrm{Y}}=0,426+0,0266 \mathrm{X}$ & 0,91 & $\begin{array}{l}\hat{Y} \\
\hat{\mathbf{Y}}\end{array}$ & $=6,655+0,1490 \mathrm{X}$ & 0,99 \\
\hline B. pilosa & $\hat{Y}=1,536+0,0771 \mathrm{X}$ & 0,97 & $\begin{array}{l}\hat{Y} \\
\hat{Y}\end{array}$ & $=12,718+0,1467 \mathrm{X}$ & 0,90 \\
\hline D. tortuosum & $\mathrm{Y}=5,812+0,1825 \mathrm{X}$ & 0,99 & Y & $=9,5162+0,5034 \mathrm{X}$ & 0,99 \\
\hline
\end{tabular}

(1) Biótipo resistente e sensível aos herbicidas inibidores da enzima ALS. * Significativo pelo teste t a 5\% de probabilidade de erro.

formação de propágulos, D. tortuosum apenas apresentou maior MST em relação a $B$. pilosa, justamente quando cultivado em solo que recebeu a dose de $\mathrm{P}$ recomendada com base na cultura do feijão $\left(24,00 \mathrm{mg} \mathrm{dm}^{-3}\right)$.
Como visto para a avaliação da MSR, os biótipos de $E$. heterophylla apresentaram a menor produção de MST entre as plantas daninhas, com exceção dos tratamentos que receberam as duas menores doses de $\mathrm{P}\left(0,0\right.$ e $\left.12,00 \mathrm{mg} \mathrm{dm}^{-3}\right)$ no início do florescimento, 
e não diferiram quanto a MST das plantas de $B$. pilosa. Também não houve diferenças entre os biótipos de E. heterophylla para essa avaliação. A partir da dose de $\mathrm{P}$ de $24,00 \mathrm{mg} \mathrm{dm}^{-3}$ na avaliação do início do florescimento e em todas as doses de $\mathrm{P}$ no final da formação de propágulos, o feijão sempre apresentou menor MST em comparação com as espécies daninhas $D$. tortuosum e B. pilosa (Quadro 4). Isso indica que o incremento no fornecimento de $\mathrm{P}$, caso não haja manejo adequado dessas plantas daninhas, pode favorecê-las em detrimento da cultura do feijão. Observou-se resposta à adição de $\mathrm{P}$, quanto ao acúmulo de MST, para todas as espécies (Quadro 3); no entanto, as maiores respostas foram observadas para as plantas de D. tortuosum e soja e para B. pilosa somente no final da formação de propágulos.

Corroborando esses resultados, Martins \& Pitelli (2000) constataram que a aplicação de $50 \mathrm{mg} \mathrm{dm}^{-3}$ de $\mathrm{P}$ ocasionou incremento de $859,2 \%$ na massa seca de plantas de Brachiaria plantaginea. Hoveland et al. (1976) descreveram que plantas D. tortuosum apresentaram redução de $78 \%$ na massa seca, quando submetidas à baixa adubação de $\mathrm{P}\left(22 \mathrm{~kg} \mathrm{ha}^{-1}\right)$, do que comparada ao maior nível de adubação $\left(90 \mathrm{~kg} \mathrm{ha}^{-1}\right)$. Esses resultados evidenciam que a correta adubação e o manejo de plantas daninhas são fundamentais na atenuação da competição sofrida pelas culturas.

No início do florescimento, não foi constatada diferença quanto ao teor de $\mathrm{P}$ nos tecidos vegetais de todas as espécies avaliadas, independentemente do nível de $\mathrm{P}$ aplicado, apresentando teor médio de $\mathrm{P}$ de $0,19 \mathrm{dag}^{\mathrm{kg}}{ }^{-1}$. Em relação à avaliação realizada no final da formação de propágulos, o feijão foi a espécie que apresentou o maior teor de $\mathrm{P}$ em seus tecidos, apenas não diferindo do encontrado para os biótipos de E. hetrophylla na dose de P de
$24,00 \mathrm{mg} \mathrm{dm}^{-3}$ e de D. tortuosum na dose de $\mathrm{P}$ de $48,00 \mathrm{mg} \mathrm{dm}^{-3}$ (Quadro 5). Todavia, Ugen et al. (2002) observaram menor concentração de P na massa seca de plantas de feijão (3,2 a $\left.3,8 \mathrm{mg} \mathrm{g}^{-1}\right) \mathrm{em}$ comparação à das plantas daninhas Bidens pilosa $\left(3,7\right.$ a $\left.4,1 \mathrm{mg} \mathrm{g}^{-1}\right)$, Galinsoga parviflora $(4,0$ a $\left.4,8 \mathrm{mg} \mathrm{g}^{-1}\right)$ e Solanum nigrum (4,0 a 4,5 mg g-1). B. pilosa e soja foram as espécies que menos concentraram P em seus tecidos. Deibert \& Utter (1989) encontraram teores de $\mathrm{P}$ em plantas de soja, variando de 2,7 a $3,1 \mathrm{~g} \mathrm{~kg}^{-1}$, no início do florescimento, de 2,4 a $2,6 \mathrm{~g} \mathrm{~kg}^{-1}$, no enchimento de grãos, e de 5,0 a 5,8 $\mathrm{g} \mathrm{kg}^{-1}$, na maturação fisiológica dos grãos.

Neste trabalho, o feijão apresentou, em média, teor de $\mathrm{P}$ três vezes maior que o observado para a soja. Dentre as plantas daninhas, os biótipos de $E$. heterophylla apresentaram maior teor de $\mathrm{P}$ até à dose de 24,00 $\mathrm{mg} \mathrm{dm}^{-3}$; entretanto, na maior dose $\left(48,00 \mathrm{mg} \mathrm{dm}^{-3}\right)$, D. tortuosum apresentou maior teor de $\mathrm{P}$ em seus tecidos, sendo também a única espécie que apresentou resposta a essa característica, porém somente com a adição de alta concentração de $\mathrm{P}$ ao solo, o que caracteriza um "consumo de luxo", quando há alta disponibilidade desse elemento no solo.

Em trabalho realizado por Pitelli et al. (1983), constatou-se que plantas de Cyperus rotundus acumularam maior teor de $\mathrm{P}$ em sua massa seca da parte aérea $\left(0,41\right.$ a $\left.0,42 \mathrm{dag} \mathrm{kg}^{-1}\right)$ do que plantas de soja $\left(0,20\right.$ a $\left.0,22 \mathrm{dag} \mathrm{kg}^{-1}\right)$. Souza et al. (1999), analisando os teores de nutrientes presentes na massa seca da parte aérea de 17 espécies de plantas daninhas, observaram maiores teores de $\mathrm{P}$ nas espécies E. heterophylla $\left(0,70 \mathrm{dag} \mathrm{kg}^{-1}\right)$ e $B$. pilosa $\left(0,47 \mathrm{dag} \mathrm{kg}^{-1}\right)$. Qasem \& Hill (1993) encontraram maior concentração de $\mathrm{P}$ nos tecidos das plantas daninhas Chenopodium album e Senecio vulgaris do que em plantas de tomate.

Quadro 4. Massa seca total (MST) das culturas do feijão e da soja e de espécies de plantas daninhas, submetidas a quatro níveis de fósforo na semeadura

\begin{tabular}{|c|c|c|c|c|c|c|c|c|}
\hline \multirow{3}{*}{ Espécie } & \multicolumn{4}{|c|}{$\begin{array}{c}\text { MST (g planta-1) } \\
\text { (Início do florescimento) }\end{array}$} & \multicolumn{4}{|c|}{$\begin{array}{l}\text { MST (g planta }{ }^{-1} \text { (Final da } \\
\text { formação de propágulos) }\end{array}$} \\
\hline & \multicolumn{8}{|c|}{ Dose de $P\left(\mathrm{mg} \mathrm{dm}^{-3}\right)$} \\
\hline & 0,00 & 12,00 & 24,00 & 48,00 & 0,00 & 12,00 & 24,00 & 48,00 \\
\hline P. vulgaris & $1,22 \mathrm{~b}$ & $1,86 \mathrm{~b}$ & $2,62 \mathrm{~b}$ & $4,42 \mathrm{~d}$ & $3,06 \mathrm{c}$ & $4,88 \mathrm{c}$ & $8,73 \mathrm{c}$ & $10,42 \mathrm{c}$ \\
\hline G. $\max$ & $4,65 \mathrm{a}$ & $7,04 \mathrm{a}$ & 8,98 a & $13,48 \mathrm{~b}$ & $8,16 \mathrm{ab}$ & $16,04 \mathrm{~b}$ & $23,37 \mathrm{ab}$ & $32,12 \mathrm{a}$ \\
\hline E. heterophylla $(\mathrm{r})^{(1)}$ & $0,32 \mathrm{~b}$ & $0,76 \mathrm{~b}$ & $0,54 \mathrm{~b}$ & $2,47 \mathrm{~d}$ & $4,62 \mathrm{bc}$ & $5,87 \mathrm{c}$ & $8,94 \mathrm{c}$ & $17,51 \mathrm{~b}$ \\
\hline E. heterophylla $(\mathrm{s})^{(1)}$ & $0,16 \mathrm{~b}$ & $1,02 \mathrm{~b}$ & $0,82 \mathrm{~b}$ & $2,79 \mathrm{~d}$ & $4,33 \mathrm{bc}$ & $5,70 \mathrm{c}$ & $8,94 \mathrm{c}$ & $16,23 \mathrm{~b}$ \\
\hline B. pilosa & $0,95 \mathrm{~b}$ & $1,94 \mathrm{~b}$ & $8,97 \mathrm{a}$ & $7,54 \mathrm{c}$ & 9,76 a & $18,89 \mathrm{ab}$ & $22,35 \mathrm{~b}$ & $34,44 \mathrm{a}$ \\
\hline D. tortuosum & $5,47 \mathrm{a}$ & $7,43 \mathrm{a}$ & $10,30 \mathrm{a}$ & $16,88 \mathrm{a}$ & $10,77 \mathrm{a}$ & $21,94 \mathrm{a}$ & $27,11 \mathrm{a}$ & $33,94 \mathrm{a}$ \\
\hline
\end{tabular}

(1) Biótipo resistente e sensível aos herbicidas inibidores da enzima ALS. Médias seguidas de letras iguais, na coluna, não diferem entre si pelo teste de Tukey a $5 \%$. 
Verificou-se que, na fase do início do florescimento, a quantidade de $\mathrm{P}$ presente em plantas de soja foi maior que a observada em plantas de feijão; todavia, no final da formação de propágulos, a soja somente apresentou maior teor de $\mathrm{P}$ em seus tecidos em relação ao feijão, quando ambos foram cultivados em solo que recebeu o dobro da dose de $\mathrm{P}$ recomendada com base na cultura do feijão $\left(48,00 \mathrm{mg} \mathrm{dm}^{-3}\right)$ (Quadro 6). Dentre as plantas daninhas, no início do florescimento, $D$. tortuosum foi a espécie que apresentou maior quantidade de $\mathrm{P}$ em seus tecidos, demonstrando ser a espécie daninha que mais extraiu $\mathrm{P}$ até esse período. No final de formação de propágulos, observou-se que, nos tratamentos em que não se adicionou $\mathrm{P}$ ao solo, não houve diferença significativa na quantidade de $\mathrm{P}$ presente nas espécies avaliadas, porém, quando da adição de qualquer dose, $D$. tortuosum foi a espécie que mais acumulou $\mathrm{P}$ entre todas as demais, chegando a apresentar, aproximadamente, duas vezes mais $\mathrm{P}$ do que a soja (Quadro 6). Não se observou diferença na quantidade de $\mathrm{P}$ acumulada entre os biótipos de E. heterophylla, em todas as doses de $\mathrm{P}$ e nas duas épocas de avaliação.

$\mathrm{O}$ aumento no fornecimento de $\mathrm{P}$ ocasionou incremento na quantidade desse nutriente presente nos tecidos das espécies avaliadas, entretanto, $D$. tortuosum foi a espécie que melhor respondeu a esse aumento nas duas épocas de avaliação e a soja, quando avaliada no início do florescimento (Quadro 3), o que concorda com os resultados encontrados por Martins \& Pitelli (2000).

No início do florescimento, a soja e o feijão não diferiram quanto à eficiência de suas raízes em absorver P (ERAP), independentemente da concentração desse nutriente no solo. Contudo, no final da formação de seus propágulos, o feijão

Quadro 5. Teor de fósforo na planta, encontrado nas culturas do feijão e da soja e em espécies de plantas daninhas, submetidas a quatro níveis de adubação com $\mathbf{P}$ na semeadura

\begin{tabular}{|c|c|c|c|c|c|}
\hline \multirow{2}{*}{ Espécie } & \multicolumn{4}{|c|}{ Dose de P (mg dm-3) } & \multirow{2}{*}{ Equação } \\
\hline & $\mathbf{0 , 0 0}$ & 12,00 & 24,00 & 48,00 & \\
\hline & \multicolumn{5}{|c|}{ —Teor de P (mg g $\left.{ }^{-1}\right)$ (Final da formação de propágulos) } \\
\hline P. vulgaris & $2,5 \mathrm{a}$ & $3,0 \mathrm{a}$ & $2,5 \mathrm{a}$ & $3,0 \mathrm{a}$ & $\hat{y}=\bar{y}=2,7$ \\
\hline G. $\max$ & $1,0 \mathrm{c}$ & $1,0 \mathrm{c}$ & $0,8 \mathrm{~b}$ & $1,3 \mathrm{bc}$ & $\hat{\mathrm{y}}=\overline{\mathrm{y}}=1,0$ \\
\hline E. heterophylla $(\mathrm{r})^{(1)}$ & $1,4 \mathrm{bc}$ & $1,5 \mathrm{bc}$ & $1,9 \mathrm{a}$ & $1,5 \mathrm{bc}$ & $\hat{\mathrm{y}}=\overline{\mathrm{y}}=1,6$ \\
\hline E. heterophylla $(\mathrm{s})^{(1)}$ & $1,7 \mathrm{~b}$ & $1,9 \mathrm{~b}$ & $2,0 \mathrm{a}$ & $1,7 \mathrm{~b}$ & $\hat{\mathrm{y}}=\overline{\mathrm{y}}=1,8$ \\
\hline B. pilosa & $1,0 \mathrm{c}$ & $1,2 \mathrm{c}$ & $1,1 \mathrm{~b}$ & $0,9 \mathrm{c}$ & $\hat{\mathrm{y}}=\overline{\mathrm{y}}=1,0$ \\
\hline D. tortuosum & $1,1 \mathrm{c}$ & $1,2 \mathrm{c}$ & $1,2 \mathrm{~b}$ & $3,1 \mathrm{a}$ & $\begin{array}{l}\hat{y}=1,17-0,008 x+1,32 x^{2} \\
R^{2}=0,99\end{array}$ \\
\hline
\end{tabular}

(1) Biótipo resistente e sensível aos herbicidas inibidores da enzima ALS. Médias seguidas de letras iguais, na coluna, não diferem entre si pelo teste de Tukey a $5 \%$.

Quadro 6. Quantidade total de fósforo por planta, determinada nas culturas do feijão e da soja e em espécies de plantas daninhas, submetidas a quatro níveis de adubação com $P$ na semeadura

\begin{tabular}{|c|c|c|c|c|c|c|c|c|}
\hline \multirow{4}{*}{ Espécie } & \multicolumn{8}{|c|}{ Fósforo } \\
\hline & \multicolumn{4}{|c|}{ Início do florescimento } & \multicolumn{4}{|c|}{ Final da formação de propágulos } \\
\hline & \multicolumn{8}{|c|}{ Dose de P (mg dm $\left.{ }^{-3}\right)$} \\
\hline & $\mathbf{0 , 0 0}$ & 12,00 & 24,00 & 48,00 & 0,00 & 12,00 & 24,00 & 48,00 \\
\hline & & & & $-\mathrm{mg} \mathrm{pl}$ & $\mathrm{nta}^{-1}$ & & 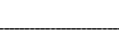 & - \\
\hline P. vulgaris & $2,04 \mathrm{~b}$ & $3,37 \mathrm{~b}$ & $4,65 \mathrm{~b}$ & $10,79 \mathrm{~b}$ & $7,31 \mathrm{a}$ & $14,77 \mathrm{bc}$ & $20,31 \mathrm{~b}$ & $31,24 \mathrm{c}$ \\
\hline G. $\max$ & $7,88 \mathrm{a}$ & $15,49 \mathrm{a}$ & $18,18 \mathrm{a}$ & $32,03 \mathrm{a}$ & $8,27 \mathrm{a}$ & $16,10 \mathrm{bc}$ & $19,58 \mathrm{~b}$ & $41,36 \mathrm{~b}$ \\
\hline E. heterophylla $(\mathrm{r})^{(1)}$ & $0,73 \mathrm{c}$ & $1,43 \mathrm{~b}$ & $1,20 \mathrm{~b}$ & $4,90 \mathrm{c}$ & $6,29 \mathrm{a}$ & $8,89 \mathrm{c}$ & $16,93 \mathrm{~b}$ & $25,90 \mathrm{c}$ \\
\hline E. heterophylla $(\mathrm{s})^{(1)}$ & $0,28 \mathrm{c}$ & $1,98 \mathrm{~b}$ & $1,72 \mathrm{~b}$ & $4,42 \mathrm{c}$ & $7,20 \mathrm{a}$ & $10,76 \mathrm{c}$ & $18,16 \mathrm{~b}$ & $28,00 \mathrm{c}$ \\
\hline B. pilosa & $2,00 \mathrm{~b}$ & $4,51 \mathrm{~b}$ & $5,84 \mathrm{~b}$ & $13,20 \mathrm{~b}$ & $9,59 \mathrm{a}$ & $21,92 \mathrm{ab}$ & $23,61 \mathrm{~b}$ & $32,65 \mathrm{bc}$ \\
\hline D. tortuosum & 6,46 a & $10,67 \mathrm{a}$ & $20,17 \mathrm{a}$ & $24,006 \mathrm{a}$ & $12,02 \mathrm{a}$ & 26,64 a & 42,16 a & 83,93 a \\
\hline
\end{tabular}

(1) Biótipo resistente e sensível aos herbicidas inibidores da enzima ALS. Médias seguidas de letras iguais, na coluna, não diferem entre si pelo teste de Tukey a $5 \%$. 
apresentou maior eficiência que a soja na retirada de P do solo (Quadro 7). D. tortuosum foi a espécie daninha que apresentou a maior ERAP no início do florescimento, quando cultivado em solo com altos teores de P.

$\mathrm{Na}$ fase final de formação de propágulos, B. pilosa e soja apresentaram a menor eficiência em extrair $\mathrm{P}$ do solo (Quadro 7). Analisando o efeito entre doses de $\mathrm{P}$ no início do florescimento quanto à ERAP somente para as plantas de $D$. tortuosum, verificouse decréscimo na ERAP, quando plantas de $D$. tortuosum foram submetidas a solo que continha a maior quantidade desse nutriente $\left(48,00 \mathrm{mg} \mathrm{dm}^{-3}\right)$. Esse resultado pode ser atribuído ao maior aumento na MSR dessa espécie na dose de $48,00 \mathrm{mg} \mathrm{dm}^{-3}$, o que não resultou em aumento na absorção de $\mathrm{P}$, na mesma proporção. Feijão e D. tortuosum tiveram aumento da ERAP com o incremento das doses de $\mathrm{P}$, na avaliação realizada no final da formação de propágulos (Quadro 7). As plantas daninhas e as plantas cultivadas diferiram quanto à habilidade de extração de nutrientes do solo e quanto à resposta na produção de massa seca em relação aos níveis de nutrientes disponíveis (Weaver \& Hamill, 1985).

A soja apresentou maior eficiência na utilização do P absorvido (EUPa) em relação ao feijão, somente na avaliação realizada no final da formação de propágulos (Quadro 8). Dentre as plantas daninhas, percebeu-se grande variação nas respostas quanto a esse índice, no entanto, $D$. tortuosum teve maior EUPa no início do florescimento em solo com baixos níveis desse elemento. No final da formação de propágulos, B. pilosa foi a espécie daninha que mostrou maior EUPa, destacando-se, principalmente, quando cultivada em solo que recebeu os maiores níveis desse nutriente (Quadro 8). As espécies avaliadas, soja e D. tortuosum, apresentaram queda na EUPa, com o aumento da dose de P aplicada em ambas as épocas de avaliação; no entanto, na segunda avaliação, a queda na soja só ocorreu para a dose mais alta de $\mathrm{P}$ aplicada (Quadro 8). A EUPa do feijão decresceu também com o incremento do nível desse nutriente no solo, porém, apenas no início do florescimento. As demais espécies não mostraram variações na EUPa com a alteração do $\mathrm{P}$ no solo. Percebeu-se (Quadro 8) que a soja utilizou melhor o $\mathrm{P}$ absorvido após o florescimento e o feijão antes desse estádio fenológico.

Em relação ao uso eficiente do $\mathrm{P}$ disponível (UEPd), a soja apresentou maior valor comparada ao feijão nas duas avaliações realizadas e em todos os níveis de $\mathrm{P}$ aplicados no solo, sendo, assim, a cultura que melhor utilizou o $\mathrm{P}$ disponível no solo na conversão de massa seca (Quadro 9). Deibert \& Utter (1989) verificaram absorção de P em plantas de soja no início do florescimento e no enchimento de grãos variando de 1,5 a $2,1 \mathrm{~kg} \mathrm{ha}^{-1}$ e de 12,8 a $14,7 \mathrm{~kg} \mathrm{ha}^{-1}$, respectivamente. Os biótipos de $E$. heterophylla apresentaram o menor UEPd com o incremento do nível de $\mathrm{P}$ no solo no início do florescimento. D. tortuosum e soja mostraram maior UEPd na fase de formação de seus propágulos, quando cultivados em solo que recebeu as doses intermediárias desse nutriente $(24,00$ e 48,00 $\mathrm{mg} \mathrm{dm}^{-3}$ ) (Quadro 9).

A soja apresentou maior percentagem de recuperação do $\mathrm{P}$ aplicado (PRPA) em relação ao feijão, somente na avaliação feita no início do florescimento (Quadro 10). Soja e D. tortuosum foram as espécies que mais absorveram o $\mathrm{P}$ aplicado nessa avaliação; contudo, na fase de formação de propágulos, $D$. tortuosum apresentou-se superior às demais espécies, chegando a absorver quase $40 \%$ do $\mathrm{P}$ aplicado ao solo na maior dose $\left(48,00 \mathrm{mg} \mathrm{dm}^{-3}\right)$ (Quadro 10). Os biótipos de E. heterophylla apresentaram o menor PRPA, entre todas as espécies seguidos pelo feijão. O PRPA variou conforme o nível de $\mathrm{P}$ no solo nas espécies $D$.

Quadro 7. Eficiência das raízes na absorção de fósforo (ERAP) das culturas do feijão e da soja e de espécies de plantas daninhas, submetidas a quatro níveis de adubação com $P$ na semeadura

\begin{tabular}{|c|c|c|c|c|c|c|c|c|c|c|}
\hline \multirow{4}{*}{ Espécie } & \multicolumn{10}{|c|}{ Fósforo } \\
\hline & \multicolumn{5}{|c|}{ Início do florescimento } & \multicolumn{5}{|c|}{ Final da formação de propágulos } \\
\hline & \multicolumn{10}{|c|}{ Dose de $P\left(\mathrm{mg} \mathrm{dm}^{-3}\right)$} \\
\hline & 0,00 & 12,00 & 24,00 & 48,00 & Equação & 0,00 & 12,00 & 24,00 & 48,00 & Equação \\
\hline P. vulgaris & 5,27 a & $4,66 \mathrm{~b}$ & $4,31 \mathrm{bc}$ & 5,85 abc & $\hat{\mathrm{y}}=\overline{\mathrm{y}}=5,02$ & 11,65 a 1 & 11,20 a & $12,04 \mathrm{a}$ & 14,46 a & $\begin{aligned} & =11,6-0,012 \mathrm{x}+0,00013 \times 2 \\
\mathrm{R}^{2} & =0,99\end{aligned}$ \\
\hline G. $\max$ & 5,35 a & $6,88 \mathrm{ab}$ & $6,54 \mathrm{~b}$ & $7,23 \mathrm{ab}$ & $\hat{y}=\bar{y}=6,25$ & $3,16 \mathrm{c}$ & $3,08 \mathrm{~d}$ & $2,71 \mathrm{c}$ & $4,10 \mathrm{~d}$ & $\hat{\mathrm{y}}=\overline{\mathrm{y}}=3,26$ \\
\hline E. heterophylla ${ }^{(1)}$ & $5,64 \mathrm{a}$ & $4,74 \mathrm{~b}$ & $5,56 \mathrm{bc}$ & $4,27 \mathrm{bc}$ & $\hat{\mathrm{y}}=\overline{\mathrm{y}}=5,05$ & $5,31 \mathrm{bc}$ & c $6,07 \mathrm{~cd}$ & $7,74 \mathrm{~b}$ & $7,01 \mathrm{~cd}$ & $\hat{\mathrm{y}}=\overline{\mathrm{y}}=6,53$ \\
\hline E. heterophylla $(\mathrm{s})^{(1)}$ & $4,68 \mathrm{a}$ & $4,61 \mathrm{~b}$ & $6,15 \mathrm{bc}$ & $3,89 \mathrm{c}$ & $\hat{\mathrm{y}}=\overline{\mathrm{y}}=4,83$ & $7,69 \mathrm{~b}$ & $8,89 \mathrm{~b}$ & $8,41 \mathrm{~b}$ & $10,53 \mathrm{~b}$ & $\hat{\mathrm{y}}=\overline{\mathrm{y}}=8,88$ \\
\hline B. pilosa & $4,97 \mathrm{a}$ & $7,68 \mathrm{ab}$ & $3,33 \mathrm{c}$ & $4,39 \mathrm{bc}$ & $\hat{\mathrm{y}}=\overline{\mathrm{y}}=5,09$ & $2,96 \mathrm{c}$ & $5,33 \mathrm{~cd}$ & $3,62 \mathrm{c}$ & $4,21 \mathrm{~d}$ & $\hat{\mathrm{y}}=\overline{\mathrm{y}}=4,03$ \\
\hline D. tortuosum & $5,73 \mathrm{a}$ & 8,12 a 1 & $10,10 \mathrm{a}$ & $7,53 \mathrm{a}$ & $\begin{array}{l}\hat{y}=5,6+0,103 x-0,00053 x^{2} \\
R^{2}=0,97\end{array}$ & $5,68 \mathrm{bc}$ & c $7,29 \mathrm{bc}$ & $7,96 \mathrm{~b}$ & $10,06 \mathrm{bc}$ & $\begin{array}{l}\hat{\mathrm{y}}=5,78+0,036 \mathrm{X}-0,00005 \mathrm{X}^{2} \\
\mathrm{R}^{2}=0,99\end{array}$ \\
\hline
\end{tabular}

(1) Biótipo resistente e sensível aos herbicidas inibidores da enzima ALS. Médias seguidas de letras iguais, na coluna, não diferem entre si pelo teste de Tukey a $5 \%$. 
tortuosum (início do florescimento) e B. pilosa (formação de propágulos) (Quadro 10), aumentando para a primeira e diminuindo para a segunda.
Os resultados obtidos evidenciam a alta capacidade de competição por nutrientes, particularmente $\mathrm{P}$, que a cultura da soja apresenta.

Quadro 8. Eficiência na utilização do fósforo absorvido (EUPa) das culturas do feijão e da soja e de espécies de plantas daninhas, submetidas a quatro níveis de $\mathbf{P}$ na semeadura

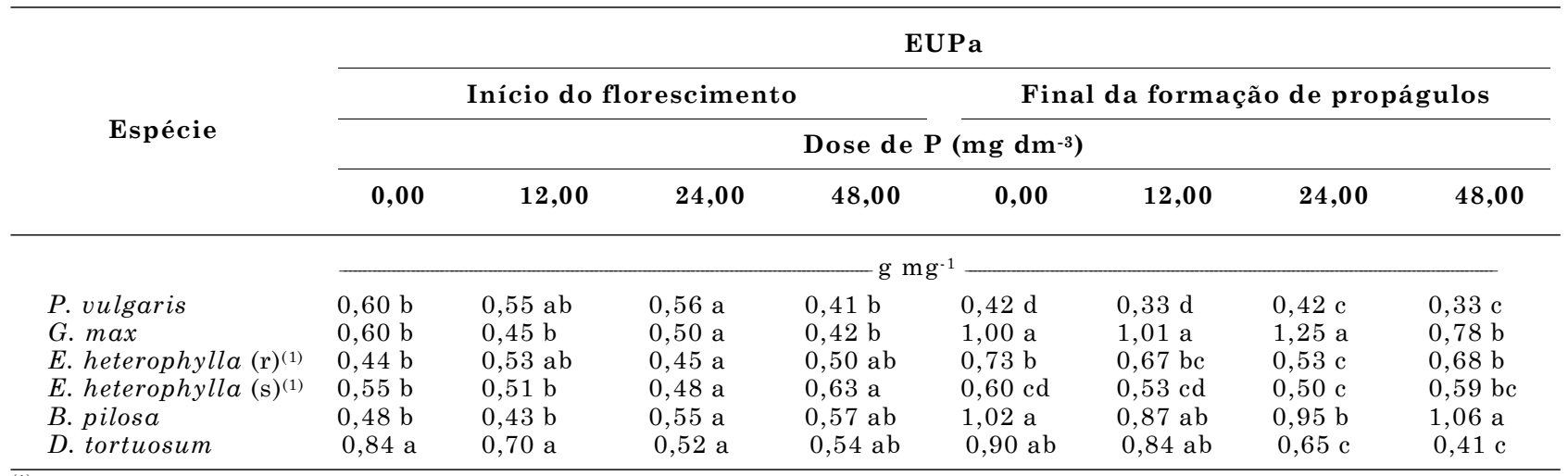

(1) Biótipo resistente e sensível aos herbicidas inibidores da enzima ALS. Médias seguidas de letras iguais, na coluna, não diferem entre si pelo teste de Tukey a $5 \%$.

Quadro 9. Uso eficiente do fósforo disponível (UEPd) das culturas do feijão e da soja e de espécies de plantas daninhas, submetidas a quatro níveis de $\mathbf{P}$ na semeadura

\begin{tabular}{|c|c|c|c|c|c|c|c|c|}
\hline \multirow{4}{*}{ Espécie } & \multicolumn{8}{|c|}{ UEPd } \\
\hline & \multicolumn{4}{|c|}{ Início do florescimento } & \multicolumn{4}{|c|}{ Final da formação de propágulos } \\
\hline & \multicolumn{8}{|c|}{ Dose de $P\left(\mathrm{mg} \mathrm{dm}^{-3}\right)$} \\
\hline & $\mathbf{0 , 0 0}$ & 12,00 & 24,00 & 48,00 & $\mathbf{0 , 0 0}$ & 12,00 & 24,00 & 48,00 \\
\hline & \multicolumn{8}{|c|}{$\mathrm{g} \mathrm{mg}^{-1}$} \\
\hline P. vulgaris & $0,12 \mathrm{a}$ & $0,11 \mathrm{~b}$ & $0,13 \mathrm{bc}$ & $0,12 \mathrm{bc}$ & $0,36 \mathrm{c}$ & $0,33 \mathrm{c}$ & $0,46 \mathrm{~b}$ & $0,34 \mathrm{~b}$ \\
\hline G. $\max$ & $0,46 \mathrm{a}$ & $0,43 \mathrm{a}$ & $0,46 \mathrm{a}$ & $0,36 \mathrm{a}$ & $0,96 \mathrm{~b}$ & $1,07 \mathrm{~b}$ & $1,28 \mathrm{a}$ & $1,05 \mathrm{a}$ \\
\hline E. heterophylla $(\mathrm{r})^{(1)}$ & $0,03 \mathrm{~b}$ & $0,04 \mathrm{~b}$ & $0,03 \mathrm{c}$ & $0,06 \mathrm{c}$ & $0,54 \mathrm{c}$ & $0,40 \mathrm{c}$ & $0,49 \mathrm{~b}$ & $0,57 \mathrm{~b}$ \\
\hline E. heterophylla $(\mathrm{s})^{(1)}$ & $0,02 \mathrm{~b}$ & $0,06 \mathrm{~b}$ & $0,04 \mathrm{c}$ & $0,07 \mathrm{c}$ & $0,51 \mathrm{c}$ & $0,39 \mathrm{c}$ & $0,49 \mathrm{~b}$ & $0,53 \mathrm{~b}$ \\
\hline B. pilosa & $0,09 \mathrm{ab}$ & $0,12 \mathrm{~b}$ & $0,17 \mathrm{~b}$ & 0,36 a & $1,15 \mathrm{ab}$ & $1,29 \mathrm{ab}$ & $1,22 \mathrm{a}$ & $1,13 \mathrm{a}$ \\
\hline D. tortuosum & $0,54 \mathrm{a}$ & 0,45 a & $0,53 \mathrm{a}$ & $0,45 \mathrm{a}$ & $1,27 \mathrm{a}$ & $1,50 \mathrm{a}$ & $1,48 \mathrm{a}$ & $1,11 \mathrm{a}$ \\
\hline
\end{tabular}

(1) Biótipo resistente e sensível aos herbicidas inibidores da enzima ALS. Médias seguidas de letras iguais, na coluna, não diferem entre si pelo teste de Tukey a $5 \%$.

Quadro 10. Percentagem de recuperação do fósforo aplicado (PRPA) das culturas do feijão e da soja e de espécies de plantas daninhas, submetidas a quatro níveis de $\mathbf{P}$ na semeadura

\begin{tabular}{|c|c|c|c|c|c|c|c|c|}
\hline \multirow{4}{*}{ Espécie } & \multicolumn{8}{|c|}{ P R P A } \\
\hline & \multicolumn{4}{|c|}{ Início do florescimento } & \multicolumn{4}{|c|}{ Final da formação de propágulos } \\
\hline & \multicolumn{8}{|c|}{ Dosede $P\left(\mathrm{mg} \mathrm{dm}^{-3}\right)$} \\
\hline & 12,00 & 24,00 & 48,00 & E qu a çã o & 12,00 & 24,00 & 48,00 & Equ a ção \\
\hline P. vulgaris & $3,65 \mathrm{c}$ & $3,59 \mathrm{~b}$ & $6,03 \mathrm{~b}$ & $\hat{\mathrm{y}}=\overline{\mathrm{y}}=4,22$ & $20,62 \mathrm{c}$ & $17,94 \mathrm{~b}$ & $16,51 \mathrm{~b}$ & $\hat{y}=\bar{y}=18,36$ \\
\hline G. $\max$ & 15,93 a & $14,22 \mathrm{a}$ & 16,67 a & $\hat{y}=\bar{y}=17,29$ & $21,62 \mathrm{bc}$ & $15,62 \mathrm{~b}$ & $22,85 \mathrm{~b}$ & $\hat{\mathrm{y}}=\overline{\mathrm{y}}=20,02$ \\
\hline E. heterophylla $(\mathrm{r})^{(1)}$ & $1,94 \mathrm{c}$ & $0,65 \mathrm{~b}$ & $2,88 \mathrm{~b}$ & $\hat{y}=\bar{y}=1,82$ & $7,17 \mathrm{~d}$ & $14,68 \mathrm{~b}$ & $13,53 \mathrm{~b}$ & $\hat{y}=\bar{y}=11,79$ \\
\hline E. heterophylla $(\mathrm{s})^{(1)}$ & $4,69 \mathrm{c}$ & $1,98 \mathrm{~b}$ & $2,85 \mathrm{~b}$ & $\hat{\mathrm{y}}=\overline{\mathrm{y}}=3,17$ & $9,82 \mathrm{~d}$ & $15,13 \mathrm{~b}$ & $14,36 \mathrm{~b}$ & $\hat{\mathrm{y}}=\overline{\mathrm{y}}=13,11$ \\
\hline B. pilosa & $6,92 \mathrm{bc}$ & $5,29 \mathrm{~b}$ & $7,73 \mathrm{~b}$ & $\hat{y}=\bar{y}=6,65$ & $34,02 \mathrm{ab}$ & $18,92 \mathrm{~b}$ & $15,92 \mathrm{~b}$ & $\begin{array}{l}\hat{y}=95,148 \mathrm{D}^{-0,5494} \\
\mathrm{R}^{2}=0,93\end{array}$ \\
\hline D. tortuosum & $11,62 \mathrm{~b}$ & $18,91 \mathrm{a}$ & $17,53 \mathrm{a}$ & $\begin{aligned} \hat{\mathrm{y}} & =0,972 \mathrm{D}-0,0141 \mathrm{D}^{2} \\
\mathrm{R}^{2} & =0,99\end{aligned}$ & 40,38 a & $41,61 \mathrm{a}$ & 49,63 a & $\hat{\mathrm{y}}=\overline{\mathrm{y}}=43,88$ \\
\hline
\end{tabular}

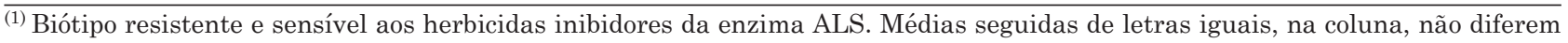
entre si pelo teste de Tukey a $5 \%$. 
Normalmente, atribui-se às plantas daninhas elevada capacidade competitiva. Em alguns casos, isso é verdadeiro, como observado neste trabalho para $D$. tortuosum. No entanto, a elevada densidade populacional que comumente caracteriza a ocorrência de plantas daninhas como E. heterophylla (que apresenta, individualmente, baixo potencial competitivo quando comparada à soja), parece ser a principal causa pelos severos danos ocasionados às culturas por competição.

\section{CONCLUSÕES}

1. A soja apresentou o maior ganho em massa seca de raízes com o incremento do fornecimento de $\mathrm{P}$, e, junto a $D$. tortuosum e B. pilosa, apresentou maior resposta à adição desse nutriente em relação ao acúmulo de massa seca total.

2. O maior teor de $\mathrm{P}$ foi constatado nas plantas de feijão, independentemente da quantidade; no entanto, $D$. tortuosum foi a única espécie a acumular mais de três vezes esse nutriente, quando cultivada em solo que recebeu $48,00 \mathrm{mg} \mathrm{dm}^{-3}$ de P.

3. A soja acumulou a maior quantidade de $\mathrm{P}$ em seus tecidos na fase inicial do florescimento; todavia, D. tortuosum apresentou essa característica durante todo o ciclo.

4. Com o aumento na adição de $\mathrm{P}$ ao solo, sua eficiência na utilização quando absorvido diminuiu para D. tortuosum, soja e feijão.

5. E. heterophylla e feijão apresentaram baixo desempenho para o uso eficiente do P disponível no solo.

6. De maneira geral, nas culturas de soja e feijão, D. tortuosum mostrou-se, dentre as espécies de plantas daninhas avaliadas, a maior competidora por $\mathrm{P}$, principalmente pela sua capacidade em extrair e armazenar esse nutriente.

\section{AGRADECIMENTO}

Ao CNPq, pelo apoio financeiro.

\section{LITERATURA CITADA}

ARMSTRONG, R.D.; BROWN, R.F. \& HELYAR, K.R. The use of nitrogen, phosphorus and lime to limit the competitive ability of Aristida armata in the establishment phase. Aust. J. Agric. Res., 44:167-178, 1993.

BLACKSHAW, R.E.; BRANDT, R.N.; JANZEN, H.H.; ENTZ, T.; GRANT, C.A. \& DERKSEN, D.A. Differential response of weed species to added nitrogen. Weed Sci., 51:532-539, 2003.
BRAGA, J.M. \& DEFELIPO, B.V. Determinação espectrofotométrica do fósforo com extratos de solos e plantas. R. Ceres, 21:73-85, 1974.

CHAGAS, J.M.; BRAGA, J.M.; VIEIRA, C.; SALGADO, L.T.; NETO, A.D.; ARAÚJO, G.A.A.; ANDRADE, M.J.B.; LANA, R.M.Q. \& RIBEIRO, A.C. Feijão. In: RIBEIRO, A.C.; GUIMARÃES, P.T.G. \& ALVAREZ V., V.H., eds. Recomendação para o uso de corretivos e fertilizantes em Minas Gerais 5 ${ }^{\mathrm{a}}$ Aproximação. 1999 . 359p.

CRALLE, H.T.; FOJTASEK, T.B.; CARSON, K. H.; CHANDLER, J.M.; MILLER, T.D.; SENSEMAN, S.A.; BOVEY, R.W. \& STONE, M.J. Wheat and italian ryegrass (Lolium multiflorum) competition as affected by phosphorus nutrition. Weed Sci., 51:425-429, 2003.

DEIBERT, E.J. \& UTTER, R.A. Growth and NPK uptake by soybean cultivars in Northern U.S.A. under reduced tillage systems. Can. J. Plant Sci., 69:1101-1111, 1989.

GILBERT, B.E. \& PEMBER, F.R. Tolerance of certain weeds and grasses to toxic aluminium. Soil Sci., 39:425-429, 1975.

HOVELAND, C.S.; BUCHANAN, G.A. \& HARRIS, M.C. Response of weeds to soil phosphorus and potassium. Weed Sci., 24:194-201, 1976.

JONES, G.D.P.; JESSOP, R.S. \& BLAIR, G.J. Alternative methods for the selection of phosphorus efficiency in wheat. Field Crops Res., 30:29-40, 1992.

MARTINS, D. \& PITELLI, R.A. Efeito da adubação fosfatada e da calagem nas relações de interferência entre plantas de soja e capim-marmelada. Planta Daninha, 18:331-347, 2000.

PATTERSON, D.T. Effects of environmental stress on weed/ crop interactions. Weed Sci., 43:483-490, 1995.

PEREIRA, P.R.G. Eficiência de utilização de frações de fósforo na soja e regulação da colonização micorrízica. Viçosa. Universidade Federal de Viçosa. 1992. 174p. (Tese de Doutorado)

PITELLI, R.A.; DURIGAN, J.C. \& BENEDETTI, N.J. Estudo de competição inter e intraespecífica envolvendo Glycine max (L.) Merril e Cyperus rotundus (L.) em condições de casa de vegetação. Planta Daninha, 6:129-137, 1983.

PONCE, R.G.; ZANCADA, C.; VERDUGO, M. \& SALAS, L. Plant height as a factor in competition between nightshade and two horticultural crops (tomato and pepper). J. Hortic. Sci., 71:453-460, 1996

QASEM, J.R. \& HILL, T.A. Effects of the form of nitrogen on the growth and nutrient uptake of tomato, groundsel and fat-hen. J. Hortic. Sci., 68:161-170, 1993.

RADOSEVICH, S.; HOLT, J. \& GHERSA, C. Weed ecology Implications for management. 2. ed. New York. John Wiley \& Sons, 1997, 589p.

SANTOS, B.M.; DUSKY, J.A.; STALL, W.M.; SHILLING, D.G. \& BEWICK, T.A. Phosphorus effects on competitive of smooth pigweed (Amaranthus hybridus) and common purslane (Portulaca oleracea) with lettuce (Lactuca sativa). Weed Sci., 46:307-312, 1998. 
SHAFIQ, M.; HASSAN, A.; AHMAD, N. \& RASHID, A. Crop yields and nutrient uptake by rainfed wheat and mungbean as affected by tillage, fertilization, and weeding. J. Plant Nutr., 17:561-577, 1994.

SIDDIQI, M.Y. \& GLASS, A.D.M. Utilization index: a modified approach to the estimation and comparison of nutrient utilization efficiency in plants. J. Plant Nutr., 4:289-302, 1981.

SOUZA, L.S.; VELINI, E.D.; MAIMONI-RODELLA, R.C.S. \& MARTINS, D. Teores de macro e micronutrientes e a relação $\mathrm{C} / \mathrm{N}$ de várias espécies de plantas daninhas. Planta Daninha, 17:163-167, 1999.

TOMASO, J.M. Approaches for improving crop competitiveness through the manipulation of fertilization strategies. Weed Sci., 43:491-497, 1995.
UGEN, M.A.; WIEN, H.C. \& WORTMANN, C.S. Dry bean competitiveness with annual weeds as affected by soil nutrient availability. Weed Sci., 50:530-535, 2002.

VENGRIS, J.; COLBY, W.G. \& DRAKE, M. Plant nutrient competition between weeds and corn. Agron. J., 47:213-216, 1955.

WEAVER, S.E. \& HAMILL, A.S. Effects of soil $\mathrm{pH}$ on competitive ability and leaf nutrient content of corn (Zea mays L.) and three weed species. Weed Sci., 33:447-451, 1985.

YOUNGQUIST, J.B.; COX, P.B. \& MARANVILLE, J.W. Evaluation of alternative screening criteria for selecting nitrogen-use efficient genotypes in sorghum. Crop Sci., 32:1310-1313, 1992. 